\title{
Distribution of the coalescence times in a system of diffusion-aggregation of particle clusters in one dimension
}

\author{
Jean-Yves Fortin ${ }^{1}$ and MooYoung Choi ${ }^{2}$ \\ ${ }^{1}$ Laboratoire de Physique et Chimie Théoriques, CNRS UMR7019, Université de \\ Lorraine, F-54000 Nancy, France \\ 2 Department of Physics and Astronomy \\ Seoul National University, Seoul 08826, South Korea \\ E-mail: jean-yves.fortin@univ-lorraine.fr, mychoi@snu.ac.kr
}

\begin{abstract}
We consider the stochastic dynamics of a system of diffusing clusters of particles on a finite periodic chain. A given cluster of particles can diffuse to the right or left as a whole and merge with other clusters; this process continues until all the clusters coalesce. We examine the distribution of the cluster numbers evolving in time, by means of a general time-dependent master equation based on a Smoluchowski equation for local coagulation and diffusion processes. Further, the limit distribution of the coalescence times is evaluated when only one cluster survives.

PACS numbers: 05.10.Gg; 05.20.Dd; 05.40.-a; 61.43.Hv
\end{abstract}

\section{Introduction}

Statistical properties of coalescence and fragmentation processes have been studied in various fields of physics and chemistry [1,2], where, for example, chemical constituents react to form new molecules or fragment into elementary components. Both reactions, aggregation and fragmentation, are governed by stochastic processes. The kinetics of such processes are described by a Smoluchowski equation [3], which is a balance equation for determining the particle distribution in size or mass in the equilibrium state $[4,5]$, and can be used for example to determine the size of interstellar dust grains $[6,7]$.

The dynamical model studied here concerns the properties of aggregating clusters of particles on a one-dimensional chain, where the hard-core condition imposes that only one particle per site is allowed. Only aggregation or coagulation in the absence of fragmentation is studied. These clusters of particles diffuse, collide and merge with other clusters. The merging occurs with probability unity when the distance between two clusters vanishes, according to the rule $A \emptyset B \rightarrow \emptyset A B$ or $A B \emptyset$, where $A$ and $B$ are two clusters of particles merging into a new cluster $A B=C$ with the size given by the sum of the individual sizes of $A$ and $B$. Also, since the chain is periodic and the process symmetric, there is no internal current. We assume that the merging rate 
between clusters is independent of the cluster size. Obviously, clusters that are far away from each other will not merge before a characteristic time which depends on their distance and diffusive length. In most of the models described by Smoluchowski equations, such coagulation rates or kernels depend intrinsically on the masses or sizes of the merging components. General descriptions of the physical properties such as mass or size distributions depend indeed on the microscopic details of these kernels $[4,8,1,9]$. For instance, in the case of a gravitational system, the aggregation rate is proportional to the sum of the masses of the two constituents, eventually with some exponents, whereas in the case of branched polymerization, they are proportional to the product of the molecular weights. Many models can be solved exactly with the help of a generating function $[4,10,11]$, which contains the information on distribution moments. The process of coalescence we consider here is irreversible and produces a single giant cluster within a finite time, which is comparable with gelation phenomena [12, 13]. This phenomena has application in the dynamics of random cellular structures such as coarsening of soap froth or bubbles in two dimensions, or evolution of magnetic domains associated with boundaries $[14,15]$. In this paper, we focus on the universal properties of the coalescence times, in terms of extreme value statistics and scaling behavior in the large-size limit.

The paper is organized as follows: In section 2, we study the local distributions of cluster sizes, the time evolution of which is governed by a stochastic master equation. The generating function for this equation allows us to compute the decreasing concentration of clusters as a function of time, and we compare the results with those obtained via numerical analysis of the stochastic process. In section 3, we consider the distribution of coalescence times in a finite-size system. We show numerically that the limit distribution described by a scaling function of the time divided by the total empty intervals length squared. A diagrammatic time expansion is also proposed in the approximation of independent intervals, and a general formula for the time distribution of an $n$-interval system is given. A comparison is made with the numerical results of the previous section, as well as modeling with a stochastic system of site diffusing heights in the presence of absorbing conditions.

\section{Master equation for the cluster size distribution}

In this section, we introduce the dynamics of clusters defined as a succession of hardcore particles surrounded by at least two empty sites on a periodic chain with $N$ sites of elementary unit length. In particular, we define the probability characterizing the size and position of the cluster on the lattice. Specifically, we write the probability for a cluster of $n(\geq 1)$ particles at time $t$ symbolically in the form

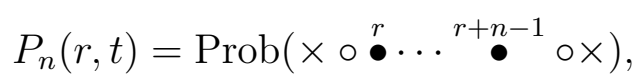

where $r$ is the site location of the first particle of the cluster. Filled and empty circles denote the sites filled with particles and empty sites, respectively while cross symbols 
$(\times)$ represent the site configuration either empty or filled with particles. The system is periodic: $P_{n}(r+N, t)=P_{n}(r, t)$. The number of particles and the number of clusters at time $t$ are given by

$$
N_{p}=\sum_{n} \sum_{r} n P_{n}(r, t) \text { and } N_{c}=\sum_{n} \sum_{r} P_{n}(r, t),
$$

respectively. The particle concentration $c \equiv N_{p} / N$ as well as the number $N_{p}$ of particles is conserved. These numbers can be estimated qualitatively via simple arguments. Assuming the simple uncorrelated process for independent particles, we have $P_{n}=N(1-c)^{2} c^{n}$ and therefore $N_{p}=\sum_{n>1} n P_{n}=N c$. Accordingly, the (mean) number of clusters is equal to $N_{c}=\sum_{n \geq 1} P_{n}=N c(1-c)$, which thus satisfies a simple semi-circle law, with the maximum cluster number at half filling $(c=1 / 2)$.

\subsection{Stochastic dynamics and the generating function}

Let us first consider the general case of fragmentation and coagulation processes. Clusters fragment or diffuse according to the following rules: At every time step $\Delta t$, a fragment of size $m(\leq n)$ can break out of the cluster from its left boundary with probability $l_{n, m}$, satisfying $\sum_{m=1}^{n} l_{n, m}=1$. Then it diffuses by one elementary step to the left at rate $1 / \tau$ and fuses eventually with another cluster. The same process also occurs on the right side with a fragment of size $m$ and probability $r_{n, m}$. In order to obtain the Smoluchowski equation, we consider different processes which contribute to the change of probability $P_{n}(r, t)$ after a small time interval $\Delta t$ and note that $\partial P_{n} / \partial t$ expresses the rate of change of the probability in the limit $\Delta t \rightarrow 0$. The first process $(a)$ is the decay or destruction of the cluster by fragmentation and diffusion:

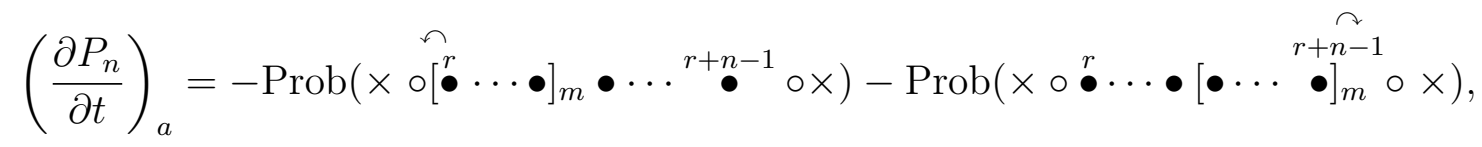

where the notation $[\bullet \cdots \bullet]_{m}$ represents a fragment of size $m$ breaking out from a cluster of larger size $n$ with probability $l_{n, m}$ or $r_{n, m}$. The right-hand side describes the rate-out process due to such decay of the cluster. The first term can thus be explicitly written as

$$
\operatorname{Prob}\left(\times \circ[\stackrel{\curvearrowleft}{r} \cdots \bullet]_{m} \bullet \cdots{ }^{r+n-1} \bullet \times\right)=\frac{1}{\tau} \sum_{m} l_{n, m} P_{n}(r)=\frac{1}{\tau} P_{n}(r)
$$

and similarly the second term. Another rate-out contribution is provided by process $(b)$, in which a fragment close to the original cluster aggregates from the left or right:

$$
\left(\frac{\partial P_{n}}{\partial t}\right)_{b}=-\operatorname{Prob}\left(\times[\bullet \cdots \bullet]_{m}^{\curvearrowright} \circ \bullet^{r} \cdot{ }^{r+n-1} \bullet \times\right)-\operatorname{Prob}\left(\times \circ \bullet^{r} \cdots^{r+n-1} \bullet \bullet[\bullet \cdots \bullet]_{m} \times\right)(5)
$$

On the other hand, positive (rate-in) contributions come from three different processes. The first one is the aggregation of clusters, labeled process $(c)$ :

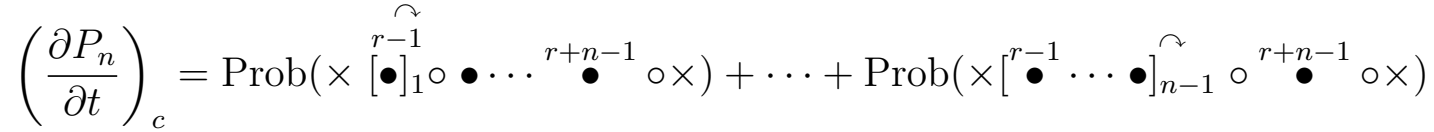

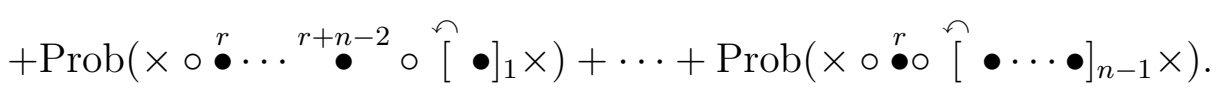


The next process $(d)$ is breaking of a fragment of size $n$ off from a larger cluster on the right or left, provided that at least two consecutive empty sites are present:

$$
\left.\left(\frac{\partial P_{n}}{\partial t}\right)_{d}=\operatorname{Prob}\left(\times[\stackrel{r-1}{\bullet} \cdots \bullet]_{n}^{\curvearrowright} \stackrel{r+n-1}{\circ} \circ \times\right)+\operatorname{Prob}(\times \circ \stackrel{r}{\circ} \stackrel{\curvearrowleft}{[} \bullet \cdots \bullet]_{n} \times\right) \text {. }
$$

Finally, the cluster can also originate from the fragmentation of a bigger cluster, which we call process $(e)$ :

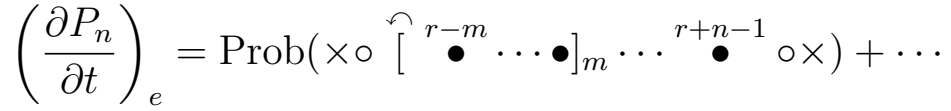

$$
\begin{aligned}
& +\operatorname{Prob}\left(\times \circ \bullet^{r} \ldots{ }^{r+n-1}\left[\bullet \ldots{ }^{r+n-1+m} \bullet^{\curvearrowright}\right]_{m} \circ \times\right) .
\end{aligned}
$$

Summation of all the contributions leads the master equation to take the form

$$
\begin{aligned}
\tau \frac{\partial P_{n}(r)}{\partial t}=- & 2 P_{n}(r)-P_{n}(r) \sum_{m \geq 1} P_{m}(r-m-1)-P_{n}(r) \sum_{m \geq 1} P_{m}(r+n+1) \\
& +\sum_{k=1}^{n-1} P_{n-k}(r+k) \sum_{m \geq k} P_{m}(r-m+k-1) r_{m, k}+\sum_{k=1}^{n-1} P_{n-k}(r) \sum_{m \geq k} P_{m}(r+n-k+1) l_{m, k} \\
& +\sum_{m \geq 0} P_{m+n}(r-1-m) r_{m+n, n}\left[1-\sum_{k \geq 1} P_{k}(r+n)\right] \\
& +\sum_{m \geq 0} P_{m+n}(r+1) l_{m+n, n}\left[1-\sum_{k \geq 1} P_{k}(r-k)\right] \\
& +\sum_{m \geq 1} P_{m+n}(r) r_{m+n, m}+\sum_{m \geq 1} P_{m+n}(r-m) l_{m+n, m}
\end{aligned}
$$

which includes terms linear or quadratic in $P_{n}$. We first notice that for an asymmetric process with the transition probability $l_{n, m}=\delta_{m, 1}$ and $r_{n, m}=\delta_{m, n}$, the dynamics produces clusters of various sizes, the distribution of which is critical for some values of the concentration $[16,17,18]$. We next consider the symmetric case, where only aggregation of clusters occurs with $r_{n, m}=l_{n, m}=\delta_{m, n}$. In this case, the master equation reduces to

$$
\begin{aligned}
\tau \frac{\partial P_{n}(r)}{\partial t}=- & 2 P_{n}(r)-P_{n}(r) \sum_{m \geq 1} P_{m}(r-m-1)-P_{n}(r) \sum_{m \geq 1} P_{m}(r+n+1) \\
& +\sum_{m=1}^{n-1} P_{n-m}(r+m) P_{m}(r-1)+\sum_{m=1}^{n-1} P_{n-m}(r) P_{m}(r+n-m+1) \\
& +P_{n}(r-1)\left[1-\sum_{m \geq 1} P_{m}(r+n)\right]+P_{n}(r+1)\left[1-\sum_{m \geq 1} P_{m}(r-m)\right],
\end{aligned}
$$

and the corresponding out-of-equilibrium dynamics leads in a finite time to an absorbing state consisting of a unique cluster.

To study how the cluster concentration decreases with time, it is convenient to introduce the generating function in the Fourier space:

$$
\hat{G}(x, k)=\sum_{r} e^{-i k r} \sum_{n \geq 1} x^{n} P_{n}(r)=\sum_{n \geq 1} x^{n} \hat{P}_{n}(k),
$$


where $\hat{P}_{n}(k) \equiv \sum_{r} e^{-i k r} P_{n}(r)$ is the Fourier transform, with the momentum taking the values $k=2 \pi m / N(m=0, \cdots, N-1)$. It is obvious from this definition that

$$
N_{c}=\hat{G}(1,0) \text { and } N_{p}=\left.\partial_{x} \hat{G}(x, 0)\right|_{x=1} .
$$

The generating function satisfies a master equation which incorporates all the above processes as well as the one-particle dynamics. Specifically, equation (10) is transformed into

$$
\begin{aligned}
\tau \frac{\partial}{\partial t} \hat{G}(x, k)= & -2(1-\cos k) \hat{G}(x, k)-\left(1+e^{-i k}\right) \frac{1}{N} \sum_{k^{\prime}} e^{i k^{\prime}} \hat{G}\left(x, k^{\prime}\right) \hat{G}\left(e^{-i\left(k-k^{\prime}\right)}, k-k^{\prime}\right) \\
& +\left(1+e^{i k}\right) \frac{1}{N} \sum_{k^{\prime}} e^{-i k^{\prime}} \hat{G}\left(x e^{i\left(k-k^{\prime}\right)}, k^{\prime}\right)\left[\hat{G}\left(x, k-k^{\prime}\right)-\hat{G}\left(1, k-k^{\prime}\right)\right]
\end{aligned}
$$

The conservation of the number of particles can be checked directly by computing $\partial_{t} N_{p}=\left.\partial_{t} \partial_{x} \hat{G}(x, 0)\right|_{x=1}=0$. On the other hand, the number of clusters, $N_{c}=\hat{G}(1,0)$, decreases according to

$$
\tau \frac{\partial}{\partial t} \hat{G}(1,0)=-\frac{2}{N} \sum_{k^{\prime}} e^{i k^{\prime}} \hat{G}\left(1, k^{\prime}\right) \hat{G}\left(e^{i k^{\prime}},-k^{\prime}\right),
$$

which depends on the contributions of momentum $k(\neq 0)$. In the long-time limit, we can consider the solutions for small momenta with $x=1$, and assume that $\hat{G}\left(e^{-i\left(k-k^{\prime}\right)}, k-k^{\prime}\right) \simeq \hat{G}\left(1, k-k^{\prime}\right)$. This simplifies equation (13) and yields a closed set of equations:

$$
\tau \frac{\partial}{\partial t} \hat{G}(1, k) \simeq-k^{2} \hat{G}(1, k)-\frac{2}{N} \sum_{k^{\prime}} e^{i k^{\prime}} \hat{G}\left(1, k^{\prime}\right) \hat{G}\left(1, k-k^{\prime}\right),
$$

which contains a diffusive term proportional to $k^{2}$ and a nonlinear term corresponding to the coalescence process.

A mean-field analysis of equation (15), employing the ansatz $\hat{G}(1, k)=N \delta_{k, 0} g(t)$, gives simply $g(t)=\left[g(0)^{-1}+2 t / \tau\right]^{-1}$, where $g(t)=N_{c} / N$ is the cluster density. A direct numerical evaluation of equation (15) also leads to the same behavior. Under this approximation, the cluster density decreases, after a long time, as $t^{-\nu}$ with $\nu=1$. For comparison, we solve equation (10) numerically, discretizing the time with increment $\Delta t=0.02$, to obtain the exponent $\nu=0.82$ for $N=200$ (see figure 1 ). Numerical analysis of equation (14) also gives the same scaling with an exponent close to unity.

These results are compared with those obtained via a stochastic method using the following rules: Starting from an initial random configuration of particles, we choose a site randomly. If the site is occupied by a particle, the whole cluster to which the particle belongs moves by one step on the right or left with equal probability. This cluster can merge with a neighboring cluster if they are separated by only one empty site. The time evolution of the cluster density obtained from such stochastic dynamics for $10^{4}$ initial random configurations is displayed in the inset of figure 1 . For size $N=500$, the decay exponent is estimated as $\nu=0.96$ in the power-law or long-time regime, which is in agreement with the approximate result discussed above. We also notice that in the case of pure particle coagulation, $A A \rightarrow A \emptyset$ or $A A \rightarrow \emptyset A$, where particles coalesce on 


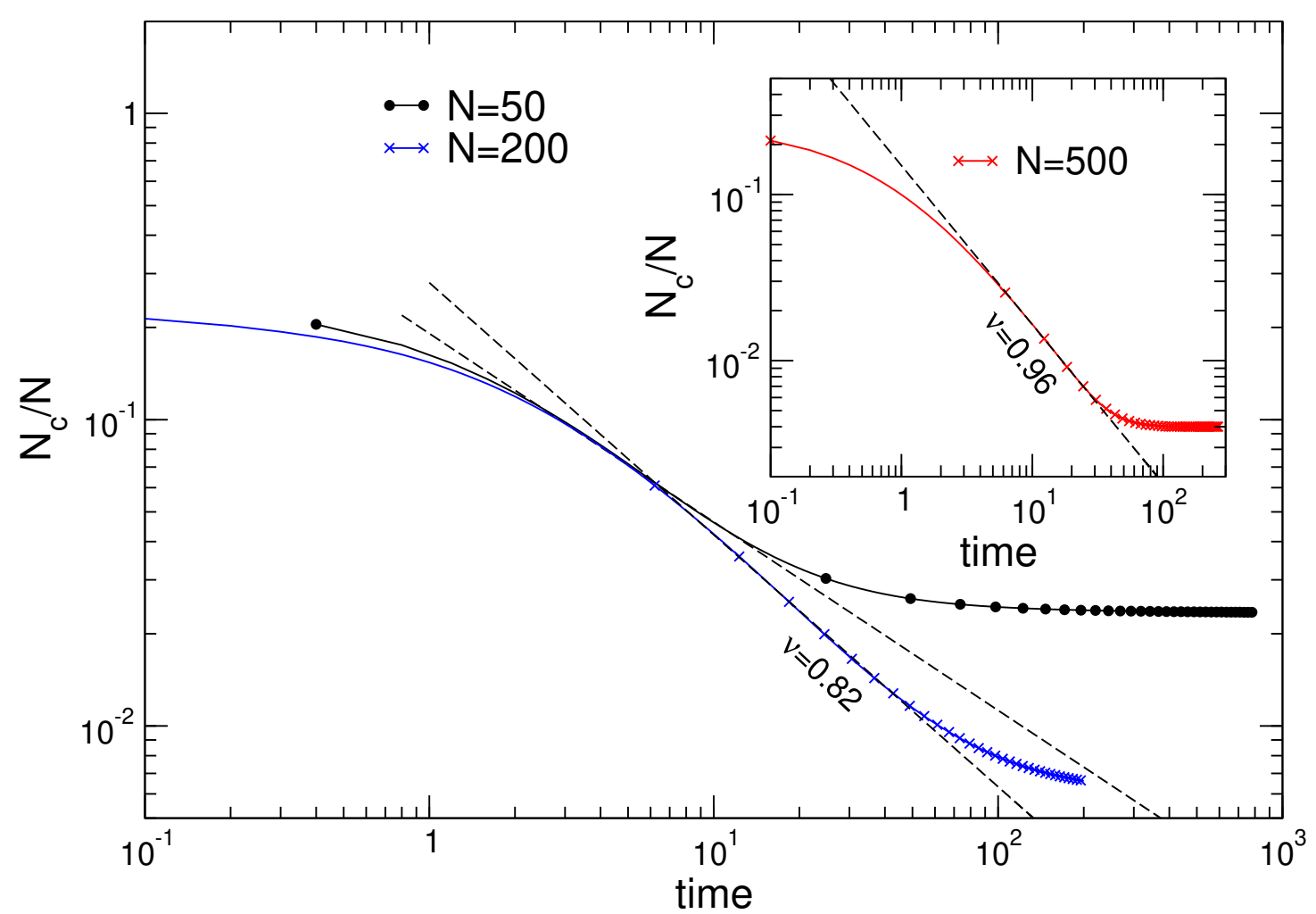

Figure 1. Time evolution of the cluster density $N_{c}$ obtained from the discrete time version of equation (10) with time increment $\Delta t=0.02$, for systems of size $N$ with concentration $c=0.5$. The decay exponent, corresponding to the slope of a dashed line, is found to be $\nu=0.82$ for $N=200$. Inset: Time evolution of the cluster density computed from the stochastic process in the system of size $N=500$ with the same particle concentration. Averages over $10^{4}$ random initial configurations are taken, with the time step $\Delta t=1 / N$. The decay exponent (given by the slope of the dashed line) is found to be $\nu=0.96$.

a single site, the exponent is given by $\nu=1 / 2$ instead [19]. In this case the particle concentration decreases and is not constant.

\subsection{Continuum limit analysis}

In this section, we examine the continuum limit in which the lattice constant $a \rightarrow 0$ and $N \rightarrow \infty$ with the length $L_{0}=N a$ finite, by introducing the space coordinate $x=r a$ and cluster size $l=n a$. All possible continuum limit should be compatible with equation (2). Distributions are rescaled according to the rule $P_{n}(r) / a^{2} \rightarrow P_{l}(x)$, and the sums are replaced by integrals. In particular, the conserved number of particles becomes a conservation relation for the total length of the clusters

$$
a N_{p}=\sum_{r} a \sum_{n} a(\text { an }) \frac{P_{n}(r)}{a^{2}} \rightarrow L_{c}=\int_{0}^{L_{0}} d x \int_{0}^{L_{c}} d l P_{l}(x) l,
$$

where $L_{c} \equiv \lim _{a \rightarrow 0} a N_{p}$ is the total length. This continuum limit transforms the problem of discrete clusters into a problem of dynamical intervals of size $l$ with probability $P_{l}(x)$ 
at location $x$. The dimensionless ratio $c=L_{c} / L_{0}(\leq 1)$ is the fraction of the lattice which is occupied by the intervals (or clusters). In accord, the number of clusters is transformed into

$$
N_{c}=\sum_{r} a \sum_{n} a \frac{P_{n}(r)}{a^{2}} \rightarrow N_{c}=\int_{0}^{L_{0}} d x \int_{0}^{L_{c}} d l P_{l}(x) .
$$

For convenience, we also define the characteristic speed $v \equiv a / \tau$ and the diffusion constant $\mathscr{D} \equiv a^{2} / \tau$, and separate equation (10) into two parts, one with all the linear diffusive terms and the other containing the quadratic terms:

$$
\begin{aligned}
\frac{1}{a^{2}} \frac{\partial P_{n}(r)}{\partial t}=\frac{1}{\tau} & {\left[\frac{P_{n}(r+1)}{a^{2}}-2 \frac{P_{n}(r)}{a^{2}}+\frac{P_{n}(r-1)}{a^{2}}\right] } \\
& +v\left[-\frac{P_{n}(r)}{a^{2}} \sum_{m=1}^{N_{p}-n} a \frac{P_{m}(r-m-1)}{a^{2}}-\frac{P_{n}(r)}{a^{2}} \sum_{m=1}^{N_{p}-n} a \frac{P_{m}(r+n+1)}{a^{2}}\right. \\
& +\sum_{m=1}^{n-1} a \frac{P_{n-m}(r+m)}{a^{2}} \frac{P_{m}(r-1)}{a^{2}}+\sum_{m=1}^{n-1} a \frac{P_{n-m}(r)}{a^{2}} \frac{P_{m}(r+n-m+1)}{a^{2}} \\
& \left.-\frac{P_{n}(r-1)}{a^{2}} \sum_{m=1}^{N_{p}-n} a \frac{P_{m}(r+n)}{a^{2}}-a \frac{P_{n}(r+1)}{a^{2}} \sum_{m=1}^{N_{p}-n} a \frac{P_{m}(r-m)}{a^{2}}\right] .
\end{aligned}
$$

In the continuum limit, the sum of the linear terms contributes in the second order in $a$ since it corresponds to a diffusive process and is therefore proportional to $\mathscr{D}$. In fact, if $v(\propto a)$ is finite, we have $\mathscr{D} / v \propto a \rightarrow 0$ and the sum of the linear terms should be negligible compared with the sum of nonlinear terms. Nevertheless, for generality, we keep the diffusive term and obtain

$$
\begin{aligned}
\frac{\partial P_{l}(x)}{\partial t}=\mathscr{D} \frac{\partial^{2} P_{l}(x)}{\partial x^{2}}+2 v\left[-P_{l}(x) \int_{0}^{L_{c}-l} d l^{\prime} P_{l^{\prime}}\left(x-l^{\prime}\right)\right. \\
\left.-P_{l}(x) \int_{0}^{L_{c}-l} d l^{\prime} P_{l^{\prime}}(x+l)+\int_{0}^{l} d l^{\prime} P_{l-l^{\prime}}\left(x+l^{\prime}\right) P_{l^{\prime}}(x)\right] .
\end{aligned}
$$

This equation includes the diffusive linear term, a term corresponding to negative contributions from clusters aggregating with the original cluster of size $l$ on the right or left, and a positive contribution coming from the aggregation of clusters of size $l^{\prime}$ and $l-l^{\prime}$.

\section{Distribution of coalescence times}

In this section, we study the distribution of the coalescence times at which all the particle clusters coalesce, starting from a random initial configuration. If we consider the coalescence of $n$ clusters, there is a finite probability that one of the $n$ intervals between two consecutive clusters vanishes at a given time, and this process continues sequentially until only one cluster or interval of size $L=(1-c) N$ survives. This coalescence time is uniquely defined for a finite system, and we expect that it scales like $L^{2}$ which is given by the diffusion length formula for a random walk process. 


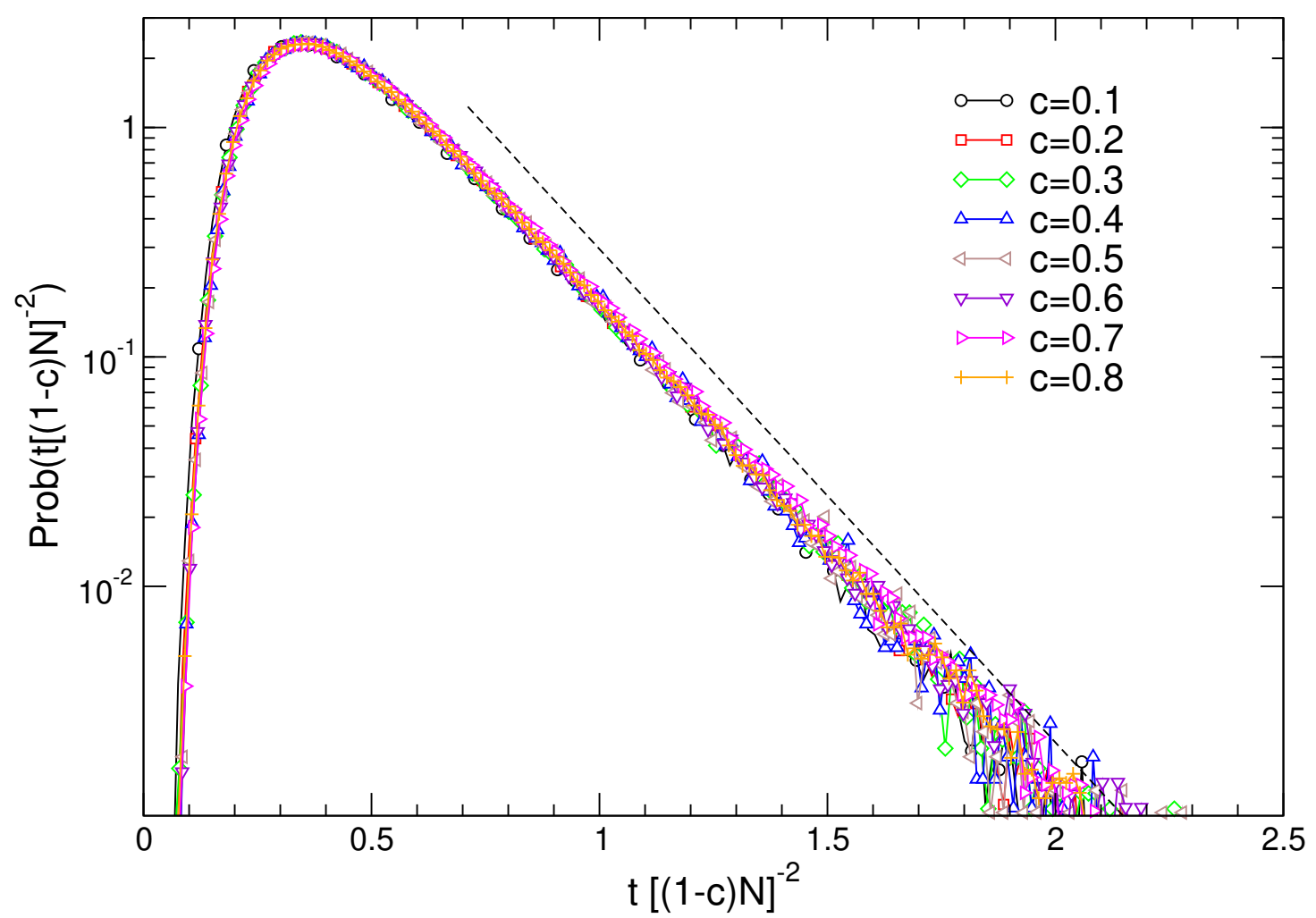

Figure 2. Coalescence time distribution for various values of the particle concentration $c$ in the system of size $N=500$. Averages over $10^{6}$ initial configurations have been taken for each concentration value. Observed is the scaling collapse with the asymptotic decay exponent found to be 4.95 (as described by the dashed line).

Figure 2 represents the plot of the coalescence time distribution rescaled with the quantity $L^{2}$; it shows clearly that all the curves collapse, regardless of the particle concentration $c$. The universal curve is found to be non-symmetric with an exponential tail whose decay exponent is approximately 4.95, close to the value $\pi^{2} / 2$ as we will demonstrate below. The problem is, in a set of initially empty intervals with different sizes that increase or decrease by diffusion, to compute the first passage time when only one interval has non-zero size. This problem can be mapped onto a $q$-state Potts model or a voter model, where particle clusters are represented by domain walls or kinks $A$ in the dual lattice between empty intervals made of holes and labeled by letters $(a, b, c, \cdots)[20,21]$, as illustrated in figure 3. Just before two domain walls merge, $A A \rightarrow A \emptyset$ or $A A \rightarrow \emptyset A$, the remaining empty hole changes its value according to the state of one of the two neighboring intervals with equal probability. This corresponds to the limit $q \rightarrow \infty$ in the Potts model since there is no annihilation process involved in the dynamics $[22,23]$. Indeed, in these theories, the probability of collision of domain walls with annihilation is equal to $1 /(q-1)$ whereas the collisions with coalescence is equal to $(q-2) /(q-1)$, yielding $q=\infty$ in our model. Moreover, the coalescence process stops when all the empty sites have the same state value.

Let us consider the case with only two intervals of sizes $h_{1}$ and $h_{2}$, which corresponds 


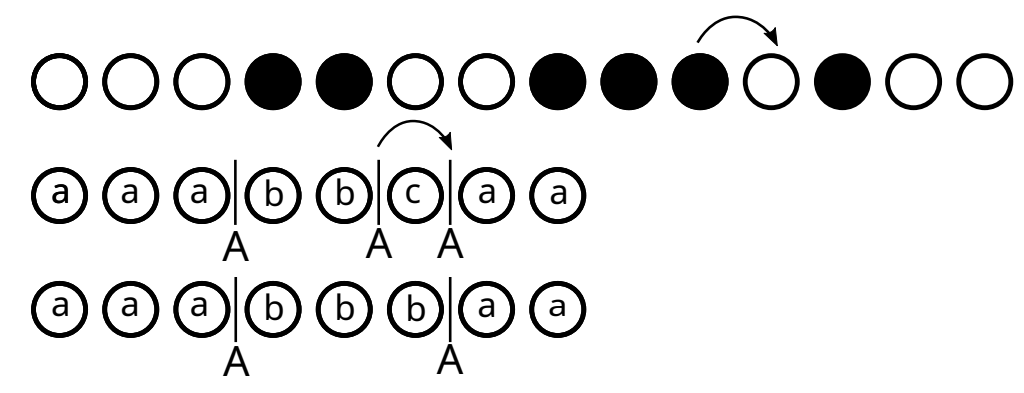

Figure 3. Representation of cluster coalescence in terms of moving domain walls (labeled $A$ ) in a $q$-state Potts model, where all the empty site intervals take different state values $(a, b, c, \cdots)$. When two clusters merge $(A A \rightarrow A)$, the hole (labeled $c$ ) changes its value according to the state of its nearest neighbors $c \rightarrow a$ or $c \rightarrow b$ (in the present case).

to two clusters of particles under the periodic conditions imposed for the lattice. When one cluster moves, $h_{1}$ and $h_{2}$ change by one unit in such a way that $h_{1} \rightarrow h_{1} \pm 1$ and $h_{2} \rightarrow h_{2} \mp 1$, subject to the constraint $h_{1}+h_{2}=L$; this reduces the problem to that with a single variable $h\left(\equiv h_{1}\right)$. A representation in terms of height dynamics is shown in figure 4(a), where the height values (lengths of the intervals or distances between clusters) decrease or increase by one unit transferred to the right or left nearest sites which have non-zero height values. The sites reaching a zero value are removed from the dynamics until one site survives with height $L$. We now consider the probability $P_{t}\left(h \mid h_{0}\right)$ that the first interval has size $h$ at time $t$, starting with a given initial condition $h_{0}\left(0<h_{0}<L\right)$ at $t=0$. This probability, abbreviated as $P_{t}(h)$ for simplicity, satisfies a differential equation of diffusion

$$
\tau \frac{\partial P_{t}(h)}{\partial t}=\frac{1}{2}\left[P_{t}(h+1)+P_{t}(h-1)-2 P_{t}(h)\right]
$$

with absorbing conditions $P_{t}(0)=P_{t}(L)=0$. This means that some of the flow of $P_{t}(h)$ is absorbed at the extremities $h=0$ or $L$ and therefore the distribution is not normalized. The discrepancy accounts for the probability of the first passage. A spectral basis for this distribution in the Fourier space is given by the set of sine functions, $\sin (k \pi h / L)$ with $k=1, \cdots, L-1$, and a simple analysis of the above differential equation leads to the expression

$P_{t}\left(h \mid h_{0}\right)=\frac{2}{L} \sum_{k=1}^{L-1} \sin \left(\frac{k \pi h}{L}\right) \sin \left(\frac{k \pi h_{0}}{L}\right) \exp \left\{-\left[1-\cos \left(\frac{k \pi}{L}\right)\right] t\right\}$.

The probability $\mathscr{P}_{t \mid h_{0}}$ of the first passage when $h$ reaches zero or $L$ relates to the time variation of the integrated distribution $[24,25]$. The integrated value is unity until the first passage time, when it begins to decrease. By the help of the summation formula $\sum_{h} \sin (k \pi h / L)=\frac{1}{2}\left[1-(-1)^{k}\right] \sin (k \pi / L)[1-\cos (k \pi / L)]^{-1}$, this probability is finally expressed as a discrete sum (with $t$ in units of $\tau$ ) [26, 27]:

$$
\mathscr{P}_{t \mid h_{0}}=-\partial_{t} \sum_{h} P_{t}\left(h \mid h_{0}\right)
$$


Distribution of coalescence times in one dimension

$$
=\frac{1}{L} \sum_{k=1}^{L-1}\left[1-(-1)^{k}\right] \sin \left(\frac{k \pi}{L}\right) \sin \left(\frac{k \pi h_{0}}{L}\right) \exp \left\{-\left[1-\cos \left(\frac{k \pi}{L}\right)\right] t\right\} .
$$

In case that $L \gg 1$ with $h_{0} / L=\alpha$ finite, this distribution has the scaling limit $t L^{2} \rightarrow t$ and $\mathscr{P}_{t \mid h_{0}} / L^{2} \rightarrow \mathscr{P}_{t \mid \alpha}$ is given by

$$
\mathscr{P}_{t \mid \alpha}=2 \pi \sum_{k=0}^{\infty}(2 k+1) \sin [(2 k+1) \pi \alpha] \exp \left[-\frac{(2 k+1)^{2}}{2} \pi^{2} t\right] .
$$

This expression is normalized and invariant under the transformation $\alpha \rightarrow 1-\alpha$ as expected, since we can replace $h_{1}$ by $h_{2}=L-h_{1}$ by symmetry. The asymptotic value is obtained for $k=0$ where $\mathscr{P}_{t \mid \alpha}$ decreases exponentially like $e^{-\pi^{2} t / 2}$. The decay coefficient $\pi^{2} / 2$ is very close to the one obtained from the numerical results in figure 2 . Nevertheless, equation (23) does not account for the numerical data of figure 2, where many interacting interval configurations are involved. We would thus like to extend this result to a system of $n$ intervals or equivalently, $n$ coalescing clusters of particles (for large $n$ ).

The next case is a system composed of three intervals $h_{1}, h_{2}$, and $h_{3}=L-h_{1}-h_{2}$. Only two variables are independent and the equation of diffusion reads

$\frac{\partial P_{t}\left(h_{1}, h_{2}\right)}{\partial t}=\frac{1}{6}\left[P_{t}\left(h_{1} \pm 1, h_{2} \mp 1\right)+P_{t}\left(h_{1} \pm 1, h_{2}\right)+P_{t}\left(h_{1}, h_{2} \pm 1\right)-6 P_{t}\left(h_{1}, h_{2}\right)\right]$,

where factor six takes into account the diffusion of each of the three clusters in the two possible directions, left or right. This equation is valid for $h_{1}$ and $h_{2}$ strictly inside the domain of existence limited by the triangular perimeter which excludes the values of $h_{1}$ and $h_{2}$ equal to zero or $L$ as well as the case $h_{1}+h_{2}=L$. When the system reaches a partial absorbing configuration where one of the three interval has a zero length (namely, $h_{1}=0$ or $h_{2}=0$ or $h_{3}=0$ ) and only two clusters survive, we should then apply equation (20) for the remaining variable. Accordingly, there is a hierarchy of diffusion equations which have been studied for an open system in the problem of coalescing walkers [28], with statistical independence of successive interval lengths and partial correlations. In their study the coalescence probability of two walkers is $p$ which corresponds here to $p=1$. The general version of equation (24) in the case of $n$ intervals with the constraint $h_{1}+\cdots+h_{n}=L$ can be written as

$$
\begin{aligned}
\frac{\partial P_{t}^{(n)}\left(\left\{h_{i}\right\}_{1 \leq i \leq n-1}\right)}{\partial t} & =\sum_{i=1}^{n-1} P_{t}^{(n+1)}\left(h_{1}, \cdots, h_{i}, \downarrow, h_{i+1}, \cdots, h_{n-1}\right) \\
+\frac{1}{n}\left(\Delta_{h_{1} h_{1}}^{2}\right. & \left.-\Delta_{h_{1} h_{2}}^{2}+\Delta_{h_{2} h_{2}}^{2}+\cdots-\Delta_{h_{n-2} h_{n-1}}^{2}+\Delta_{h_{n-1} h_{n-1}}^{2}\right) P_{t}^{(n-1)}\left(\left\{h_{i}\right\}\right),
\end{aligned}
$$

where $P_{t}^{(n)}\left(\left\{h_{i}\right\}\right)$ is the interval distribution for $n$ clusters. We have also defined the discrete Laplacian operators $\Delta_{h h} f_{h} \equiv f_{h+1}+f_{h-1}-2 f_{h}$ and mixed derivatives $2 \Delta_{h h^{\prime}} f_{h, h^{\prime}} \equiv \Delta_{h h} f_{h, h^{\prime}}+\Delta_{h^{\prime} h^{\prime}} f_{h, h^{\prime}}-f_{h+1, h^{\prime}-1}-f_{h-1, h^{\prime}+1}+2 f_{h, h^{\prime}}$. The down arrow in the term on the first line symbolizes the discrete derivative operator at $h=0$ at the corresponding position and the sum represents the multiple collision rates of two clusters coming from configurations with $n+1$ intervals [28]. The mixed derivatives account 

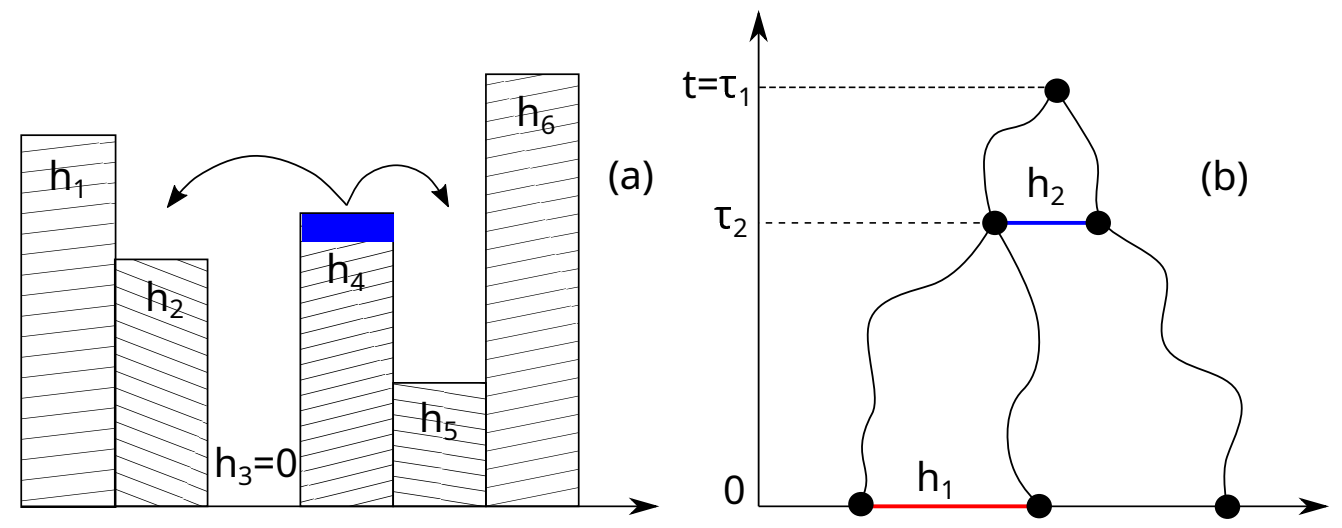

Figure 4. (a) Modelling of the interval dynamics in terms of heights. A site decreases its height value by one unit (here $h_{4} \rightarrow h_{4}-1$ ) which is transferred on the nearest site, right or left with equal probability, providing it has a non zero value. As example, site 4 can transfer some of its height (in blue color) to sites 2 or 5 , as site 3 which has zero height is non active in the dynamics. (b) Schematic time representation diagram for the coalescence of three particles or intervals under implicit periodic boundary conditions. At time $\tau_{2}$ the first two clusters coalesce and the red interval vanishes. Subsequently, at final time $\tau_{1}=t$ the remaining two clusters coalesce, reaching the absorbing state in which the blue interval vanishes.

for the particular diffusion of the intervals, which are not locally independent, as the increase of a given interval leads to the decrease of the neighboring interval on the left or right by the same amount. Instead of solving directly equation (25), we consider the diagrammatic expansion illustrated in figure 4(b) for three clusters as an example. Given an $n$-cluster or interval configuration, we first evaluate the probability that one of the $n$ intervals collapses at time $\tau_{n-1}(>0)$, leading to an $(n-1)$-interval state. The process is repeated until only one cluster survives at time $t=\tau_{n-1}+\tau_{n-2}+\cdots+\tau_{1}$. The distribution of times $t$ then gives the probability of coalescence. We also assume that the intervals are independent but satisfy statistically the global constraint $h_{1}+\cdots+h_{n}=L$, leaving $n-1$ independent variables. We finally average over all the interval size configurations to obtain the approximate distribution in the form:

$\mathscr{P}_{\text {tot }}^{(n)}(t)=\left[\int_{0}^{t} \mathscr{P}_{\tau_{n-1} \mid h_{1}} d \tau_{n-1} \int_{0}^{t-\tau_{n-1}} \mathscr{P}_{\tau_{n-2} \mid h_{2}} d \tau_{n-2} \cdots \int_{0}^{t-\tau_{n-1}-\cdots \tau_{3}} \mathscr{P}_{\tau_{2} \mid h_{n-2}} \mathscr{P}_{\tau_{1} \mid h_{n-1}} d \tau_{2}\right]_{\left\{h_{i}\right\}}$ (26)

where $[\cdots]_{\left\{h_{i}\right\}}$ stands for the average over all the interval configurations. Taking the Laplace transform leads to

$$
\overline{\mathscr{P}}_{\text {tot }}^{(n)}(s) \equiv \mathcal{L}\left(\mathscr{P}_{t}^{(n)}, s\right)=\prod_{m=2}^{n} \overline{\mathscr{P}}_{s /(m-1)}^{(m)} .
$$

The coefficients $1 /(m-1)$ take into account the fact that there is a probability $1 /(m-1)$ to choose an interval among $m-1$ independent intervals. It relates to the factor $1 / n$ in the diffusion constant in equation (25), which rescales the time for each $n$-cluster configuration. There is a symmetry coefficient $n$ ! included in the normalization factor as we have $n$ possible choices in choosing each coalescent pair of clusters in an $n$-cluster 
configuration. The Laplace transform $\overline{\mathscr{P}}_{s}^{(n)}$ can be written explicitly as

$$
\overline{\mathscr{P}}_{s}^{(n)}=\frac{1}{L} \sum_{k=1}^{L-1}\left[1-(-1)^{k}\right] \frac{\sin (k \pi / L)}{s+1-\cos (k \pi / L)} g_{n}(k),
$$

where $g_{n}(k)$ is the interval size average of the term $\sin \left(k \pi h_{0} / L\right)$ in equation $(22)$ for an $n$-interval configuration and is defined by

$$
g_{n}(k)=\frac{\sum_{\left\{h_{i} \geq 1\right\}} \delta_{L, h_{1}+\cdots+h_{n}} \sin \left(k \pi h_{1} / L\right)}{\sum_{\left\{h_{i} \geq 1\right\}} \delta_{L, h_{1}+\cdots+h_{n}}} .
$$

The summation can be performed through the use of the integral representation of the Kronecker delta function. The number of configurations is thus evaluated as

$$
\begin{aligned}
\sum_{\left\{h_{i} \geq 1\right\}} \delta_{L, h_{1}+\cdots+h_{n}} & =\sum_{\left\{h_{i} \geq 1\right\}} \int_{-\pi}^{\pi} \frac{d \theta}{2 \pi} e^{i \theta\left(L-h_{1}-\cdots-h_{n}\right)}=\int_{-\pi}^{\pi} \frac{d \theta}{2 \pi} e^{i \theta L}\left(\sum_{h \geq 1} e^{-i \theta h}\right)^{n} \\
& =\oint_{C_{1+\epsilon}} \frac{d z}{2 i \pi} \frac{z^{L-1}}{(z-1)^{n}}=\left(\begin{array}{c}
L-1 \\
n-1
\end{array}\right),
\end{aligned}
$$

where the path of the complex integral follows any circle of radius strictly larger than unity. Applying the same technique to the sine function, we obtain

$$
\begin{aligned}
\left(\begin{array}{c}
L-1 \\
n-1
\end{array}\right) g_{n}(k)= & \left\{\sum_{l=0}^{n-2}\left(\begin{array}{c}
L-1 \\
n-2-l
\end{array}\right) \frac{(-1)^{l}}{2^{l+1}} \frac{\sin \left[(1-l) \frac{k \pi}{2 L}+(l+1) \frac{\pi}{2}\right]}{\sin (k \pi / 2 L)^{l+1}}\right. \\
& \left.+(-1)^{k+1} \frac{\sin \left[(n-1) \frac{k \pi}{2 L}+(n-1) \frac{\pi}{2}\right]}{2^{n-1} \sin (k \pi / 2 L)^{n-1}}\right\},
\end{aligned}
$$

which, as $L$ grows large, approaches a finite limit independent of $L$ :

$g_{n}(k)=\sum_{l=0}^{n-2} \frac{(n-1) !}{(n-2-l) !} \frac{(-1)^{l}}{(k \pi)^{l+1}} \sin \left(\frac{l+1}{2} \pi\right)+(n-1) ! \frac{(-1)^{k+1}}{(k \pi)^{n-1}} \sin \left(\frac{n-1}{2} \pi\right)$.

In figure 5, the inverse Laplace transform of equation (27) is plotted for $n=8$ and $n=12$. The agreement with the scaling curve of figure 2 (we have taken $c=0.5$ for reference) is indeed manifested for large $t$.

We also compare the obtained analytical and numerical results with a model based on the stochastic process of equations (24) and (25). Specifically, we consider the diffusion of $n$ sites with non-zero random heights, where $n$ corresponds to the number of intervals, see figure 4(a). A site, say $i$, is randomly chosen $(i=1, \ldots, n)$ and its height value $h_{i}$ is decreased by one unit, while one of the two randomly chosen nearest neighbor sites with non-zero height has its value increased by the same amount. The sites whose height reaches zero value become inactive and are removed, and the number of active sites decreases. The dynamics of local height diffusion stops when the coalescence time is reached, at which all the sites are empty except one, when only a site with the maximum height remains. For $n=10$ and $n=20$ sites, the resulting distribution of times converges rapidly to the scaling curve of figure 2, with the chosen constraint $\sum_{i=1}^{n} h_{i}=100$. The average number of clusters in a finite system of concentration $c$ is 


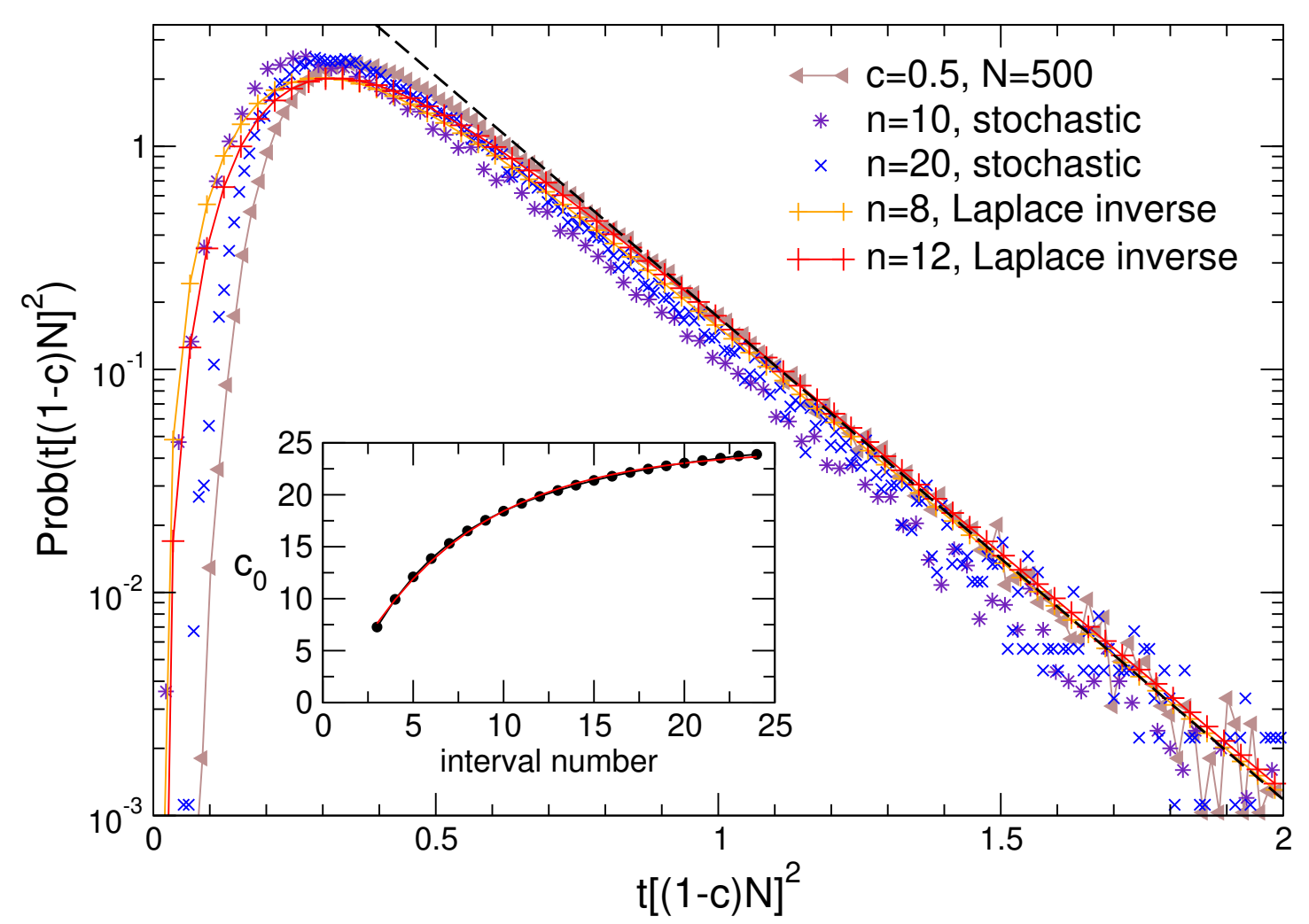

Figure 5. Coalescence time distribution. The curves with plus symbols are results from the inverse Laplace transform, equation (27). The curves with star and cross symbols are obtained from stochastic numerical analysis of diffusive heights located on each site, with $n=10$ and $n=20$ sites. Averages are performed with $2 \times 10^{5}$ initial samples, and the sum of the heights is 100 , which corresponds to $(1-c) N$. The curve of figure 2 with $c=0.5$ is given for comparison, and the dashed line is the fit of this curve in the asymptotic region $t \geq 1$. Inset: Plot of the coefficient $c_{0}$ (filled circles), see equation (35), versus the number of intervals. The red curve is an interpolation with an exponential function (see text), giving an asymptotic estimate $c_{0} \approx 24.4$.

equal to $N c(1-c)$, with the standard deviation given by $\sqrt{N} c(1-c)$, and we expect that the universal distribution should be correctly dominated by the configurations of a large number of clusters around this mean value. For example, when $c=0.5$ and $N=500$, this corresponds to $n=125$, and for $c=0.1, n=45$. Both cases give the same universal curve of figure 2 which should be achieved for a relatively large number of intervals. We can interpret the coalescence time distribution $\mathscr{P}_{t o t}^{(n)}(t)$, using the characteristic function of the survival time interval $\tau_{k}$ and writing the definition

$$
\begin{aligned}
\mathscr{P}_{\text {tot }}^{(n)}(t) & =\left\langle\delta\left(t-\tau_{1}-\tau_{2}-\cdots-\tau_{n-1}\right)\right\rangle_{\tau_{1}, \cdots, \tau_{n-1}}=\int_{-\infty}^{\infty} \frac{d \lambda}{2 \pi} e^{i \lambda t} \prod_{k=1}^{n-1}\left\langle e^{-i \lambda \tau_{k}}\right\rangle_{\tau_{k}} \\
& \simeq \int_{-\infty}^{\infty} \frac{d \lambda}{2 \pi} e^{i \lambda t} \prod_{m=2}^{n} \sum_{k \geq 1} \frac{a_{k}^{(m)}}{i \frac{\lambda}{m-1}+\omega_{k}}
\end{aligned}
$$


Distribution of coalescence times in one dimension

$$
=\int_{-\infty}^{\infty} \frac{d \lambda}{2 \pi} e^{i \lambda t} \sum_{k_{2}, \cdots, k_{n} \geq 1} \frac{a_{k_{2}}^{(2)} \cdots a_{k_{n}}^{(n)}}{\left(i \lambda+\omega_{k_{2}}\right) \cdots\left(i \frac{\lambda}{n-1}+\omega_{k_{n}}\right)},
$$

where coefficient $a_{k}^{(m)}$ and $\omega_{k} \equiv 1-\cos (\pi k / L)$ are defined from $\overline{\mathscr{P}}_{s}^{(m)}=\sum_{k \geq 1} a_{k}^{(m)}(s+$ $\left.\omega_{k}\right)^{-1}$ [see equation (28)]. The singularities of the integrand are located at $\lambda=i \omega_{k_{l}}(l-1)$ in the upper complex plane. This allows us to compute the integral by means of the residue formula, assuming that the singularities are all simple poles:

$$
\mathscr{P}_{t o t}^{(n)}(t) \simeq \sum_{l=2}^{n} \sum_{k_{2}, \cdots, k_{n} \geq 1} \frac{(l-1) a_{k_{2}}^{(2)} \cdots a_{k_{n}}^{(n)}}{\prod_{j \neq l}\left(\omega_{k_{j}}-\omega_{k_{l}} \frac{l-1}{j-1}\right)} e^{-\omega_{k_{l}}(l-1) t} .
$$

Asymptotically, the dominant contribution of $\mathscr{P}_{\text {tot }}^{(n)}(t)$ comes from $l=2$ and $k_{2}=1$, which yields an exponential decay: $\mathscr{P}_{\text {tot }}^{(n)}(t) \simeq c_{0}(n) e^{-(1-\cos (\pi / L)) t} \simeq c_{0}(n) e^{-\left(\pi^{2} / 2 L^{2}\right) t}$. The coefficient $c_{0}$ can be extracted from equation (34) in the scaling limit $t \rightarrow t L^{2}$ :

$$
c_{0}(n)=L^{2} a_{1}^{(2)} \prod_{m=3}^{n}\left[\sum_{k=1}^{L-1} \frac{a_{k}^{(m)}}{\omega_{k}-\omega_{1}(m-1)^{-1}}\right],
$$

with $a_{k}^{(n)}=L^{-1}\left[1-(-1)^{k}\right] \sin (k \pi / L) g_{n}(k)$. In the limit of large $L$, we have

$$
\lim _{L \rightarrow \infty} \frac{a_{k}^{(m)}}{\omega_{k}-\omega_{1}(m-1)^{-1}}=\frac{2 k}{\pi} \frac{\left[1-(-1)^{k}\right]}{k^{2}-(m-1)^{-1}} g_{m}(k),
$$

and coefficient $c_{0}$ reduces to

$$
c_{0}(n)=4 \prod_{m=3}^{n}\left[\sum_{k=1}^{\infty} \frac{2 k}{\pi} \frac{\left[1-(-1)^{k}\right]}{k^{2}-(m-1)^{-1}} g_{m}(k)\right],
$$

where $g_{m}(k)$ has been evaluated from equation (32). Since $g_{n}(k) \simeq(n-1) /(\pi k)$ for $k$ large, the sums over $k$ in equation (37) converge as each term behaves asymptotically like $k^{-2}$. We have computed $c_{0}(n)$ for several values of the interval number $n$ and plot the result in the inset of figure 5 (black circles). These data points fit well with the red line in the inset described by an exponential function $c_{0}-c_{1} e^{-c_{2} n}$ with $c_{0} \approx 24.41$, $c_{1} \approx 26.16$, and $c_{2} \approx 0.147$. The dashed line in figure 5 represents the asymptotic fit for the curve of concentration $c=0.5$ of figure 2 with $L=250$ in the region $t \geq 1$, and the intersection at the origin gives $c_{0} \simeq 24.9$, which is close to the analytical result in equation (37). It is therefore concluded that the theory describes accurately the asymptotic regime of coalescent clusters.

\section{Conclusion}

Stochastic dynamics of coalescent clusters in a finite-size system displays universal behavior which can be accounted for by a model of statistically independent intervals. This is in turn mapped onto a system of diffusing intervals or heights in one dimension, with absorbing conditions. The probability distribution of the interval size configurations follows a hierarchical descending set of diffusive equations between configurations involving successively $n, n-1, \ldots$, and 1 intervals, and whose approximate 
solution can be constructed by considering the time averaged product of successive single absorbing state probabilities. We have then used a Laplace transform to express the global distribution of coalescence times as the product of local Laplace distributions, assuming the independence of intervals but taking into account the finite size constraint for a given number of intervals. The results are corroborated quantitatively by a model of positive heights with absorbing conditions on site, where sites reaching zero height value are removed from the dynamics. In this case the universal curve is recovered numerically in the limit of large numbers of initial intervals. The asymptotic behavior of the universal curve, which follows and exponential, is well accounted for by the theory where the numerical factor and decay coefficient of the exponential can be extracted exactly. The method is less accurate near the origin where local correlations between intervals should be taken into consideration. We finally note that this problem is related to other problems involving the survival time distribution or time to complete coalescence of reactants on different geometries, such as coalescence of particles on fully-connected lattices $[29,30]$ where the limit distribution has a similar asymptotic exponential form in $e^{-2 t}$, but with a singular behavior in $t^{-7 / 2} e^{-\pi^{2} / 4 t}$ for $t$ small, with $t$ being the rescaled time proportional to the inverse of the number of sites.

\section{Acknowledgments}

This work was supported by the National Research Foundation of Korea through the Brain Pool Program (NRF-2018H1D3A2065321) and also through the Basic Science Research Program (Grant No. 2019R1F1A1046285).

\section{References}

[1] Wattis J A 2006 Physica D 222 1-20

[2] Kyprianou A E, Pagett S W and Rogers T 2018 Ann. Inst. Henri Poincaré, B $541134-1151$ (Preprint 1504.03196)

[3] Smoluchowski M 1916 Physik. Zeit. 17 557-585

[4] Warren H W 1981 J. Colloid Interface Sci. 87 204-208

[5] Dammer S M 2004 Stochastic many-particle systems with irreversible dynamics Ph.D. thesis Universität Duisburg-Essen

[6] Hayakawa H and Hayakawa S 1988 Publ. Astron. Soc. Japan 40 341-345

[7] Brilliantov N, Krapivsky P L, Bodrova A, Spahn F, Hayakawa H, Stadnichuk V and Schmidt J 2015 Proceedings of the National Academy of Sciences 112 9536-9541

[8] Botet R and Jullien R 1984 J. Phys. A 17 2517-2530

[9] Ben-Naim E and Krapivsky P L 2007 Phys. Rev. E 75011103 (Preprint 0608519 )

[10] Hayakawa H 1987 J. Phys. A 20 L801-L805

[11] Connaughton C, Rajesh R and Zaboronski O 1999 Kinetics of Cluster-Cluster Aggregation (Boca Raton: CRC) chap 16, pp 1-17

[12] Babovsky H 2006 Physica D: Nonlinear Phenomena 222 54-62

[13] Lushnikov A A 2006 Physica D: Nonlinear Phenomena 222 37-53

[14] Stavans J 1993 Reports Prog. Phys. 56 733-789

[15] Levitan B and Domany E 1997 Europhys. Lett. 38 485-490

[16] Vigil R D, Ziff R M and Lu B 1988 Phys. Rev. B 38942 
[17] Majumdar S N, Krishnamurthy S and Barma M 2000 J. Stat. Phys. 99 1-29 (Preprint 9908443)

[18] Fortin J Y 2019 Phys. Rev. E 100(5) 052108

[19] Ben-Avraham D, Burschka M A and Doering C R 1990 J. Stat. Phys. 60 695-728

[20] Sire C and Majumdar S N 1995 Phys. Rev. E 52244

[21] Fontes L R, Isopi M, Newman C M and Stein D L 2001 Phys. Rev. Lett. 87 1-4

[22] Krapivsky P L and Ben-Naim E 1997 Phys. Rev. E 56 3788-3798

[23] Ben-Naim E and Krapivsky P L 1998 J. Stat. Phys. 93 583-601

[24] Redner S 2001 First-Passage Processes (New-York: Cambridge University Press)

[25] Nagar A and Pradhan P 2003 Phys. A Stat. Mech. its Appl. 320 141-148

[26] Kac M 1945 Ann. Math. Stat. 16 62-67 URL http://projecteuclid.org/euclid.aop/1176996548

[27] Andrei N Borodin P S a 2002 Handbook of Brownian Motion - Facts and Formulae 2nd ed Probability and Its Applications (Birkhäuser Basel) page 122, appendix 1.6

[28] Fatkullin I and Vanden-Eijnden E 2003 J. Stat. Phys. 112 155-163

[29] Krapivsky P, Redner S and Ben-Naim E 2010 A Kinetic View of Statistical Physics (New-York: Cambridge University Press) page 388

[30] Turban L and Fortin J Y 2018 Journal of Physics A: Mathematical and Theoretical 51145001 